\title{
A rare case of toxic epidermal necrolysis in pregnancy
}

\author{
Reena Yadav', Astha Srivastava ${ }^{1 *}$, Preeti Pawar', Vibhu Mendiratta ${ }^{2}$, Prerna Tayal $^{1}$
}

\begin{abstract}
${ }^{1}$ Department of Obstetrics and Gynecology, Lady Hardinge Medical College and SSKH, New Delhi, India
${ }^{2}$ Department of Dermatology, Lady Hardinge Medical College and SSKH, New Delhi, India
\end{abstract}

Received: 04 December 2019

Accepted: 31 December 2019

\section{*Correspondence:}

Dr. Astha Srivastava,

E-mail: astha1402@gmail.com

Copyright: () the author(s), publisher and licensee Medip Academy. This is an open-access article distributed under the terms of the Creative Commons Attribution Non-Commercial License, which permits unrestricted non-commercial use, distribution, and reproduction in any medium, provided the original work is properly cited.

\begin{abstract}
Stevens-Johnson syndrome (SJS) is a group of toxic necrolytic group of disorder of skin and mucous membrane with significant morbidity and mortality. It is a highly serious allergic reaction to medications affecting the skin and mucous membranes. Pregnant women with SJS or toxic epidermal necrolysis (TEN) are a unique subset, and both conditions can simultaneously affect the mother and fetus. It is a rare condition with a reported incidence of one case per million people per year. Till date, few cases of pregnancy with SJS/TEN have been reported. We are reporting a case of 20-year-old primigravida with $31+3$ weeks of gestation presenting with extensive toxic epidermal necrosis. Attack of SJS developing in pregnancy can be fatal because immunity is compromised. This patient was managed in our institute with involvement of multidisciplinary team and had a successful pregnancy outcome. Perinatal outcome was also good in this case.
\end{abstract}

Keywords: Pregnancy, Stevens-Johnson syndrome, Toxic epidermal necrolysis

\section{INTRODUCTION}

Stevens-Johnson syndrome (SJS) is a group of toxic necrolytic group of disorder of skin and mucous membrane with significant morbidity and mortality. SJS and TEN are considered a spectrum of the same disease. In SJS, there is $<10 \%$ of epidermal detachment and in TEN there is $>30 \%$ of epidermal detachment; with SJS/TEN overlap lying between these two extremes. ${ }^{1}$ It is a highly serious allergic reaction to medications affecting the skin and mucous membranes, characterized by severe purulent conjunctivitis, stomatitis with extensive mucosal necrosis, and purpuric macules. The pathogenesis is imperfectly understood and includes genetic factors. ${ }^{2}$ Pregnant women with SJS or TEN are a unique subset, and both conditions can simultaneously affect the mother and fetus. ${ }^{2-4}$ It is believed to be immune complex mediated reaction. It is triggered most commonly by certain drugs such as antibiotics and anti-viral agents. The common culprits are antimicrobials sulphonamide followed by nonsteroidal anti-inflammatory drugs (NSAIDs), anticonvulsant drugs, and anti-gout drugs. It is a rare condition with a reported incidence of one case per million people per year. Till date, few cases of pregnancy with SJS/TEN have been reported. We are reporting a case of 20-year-old primigravida with $31+3$ weeks of gestation presenting with extensive toxic epidermal necrosis. This patient was managed in our institute with involvement of multidisciplinary team and had a successful pregnancy outcome. Perinatal outcome was also good in this case.

\section{CASE REPORT}

A 20-year-old primigravida at 31 weeks 4 days period of gestation (POG) presented to our obstetric emergency with complaint of rashes all over the body. She was a booked case at a private clinic with regular antenatal visits and uneventful antenatal period till 29 weeks of gestation. She consulted her private practitioner at 29 
weeks POG for high grade fever and burning micturition, for which she was investigated and advised tablet Cefixime along with other supportive medications in view of positive Widal test. Although fever subsided after initiation of treatment; she continued oral Cefixime for 14 days as advised. 2 days after completion of therapy, she again developed fever with rashes all over body, with gradual development of pruritic maculo-papular eruption over trunk, limbs and face involving eyes and oral cavity. With this history she presented in obstetric emergency at Lady Hardinge Medical College. There was with no complaint of pain abdomen, leaking or bleeding per vagina and no history of allergy to drugs and food product. There was no other significant past medical and family history. Physical examination was significant for poor general condition, dehydration, fever $\left(101.2^{\circ} \mathrm{F}\right)$, pruritic morbilliform eruption all over body with involvement of hand, feet, and oral mucosa. Pedal edema was present and there was inflammation around lesions (Figure 1).

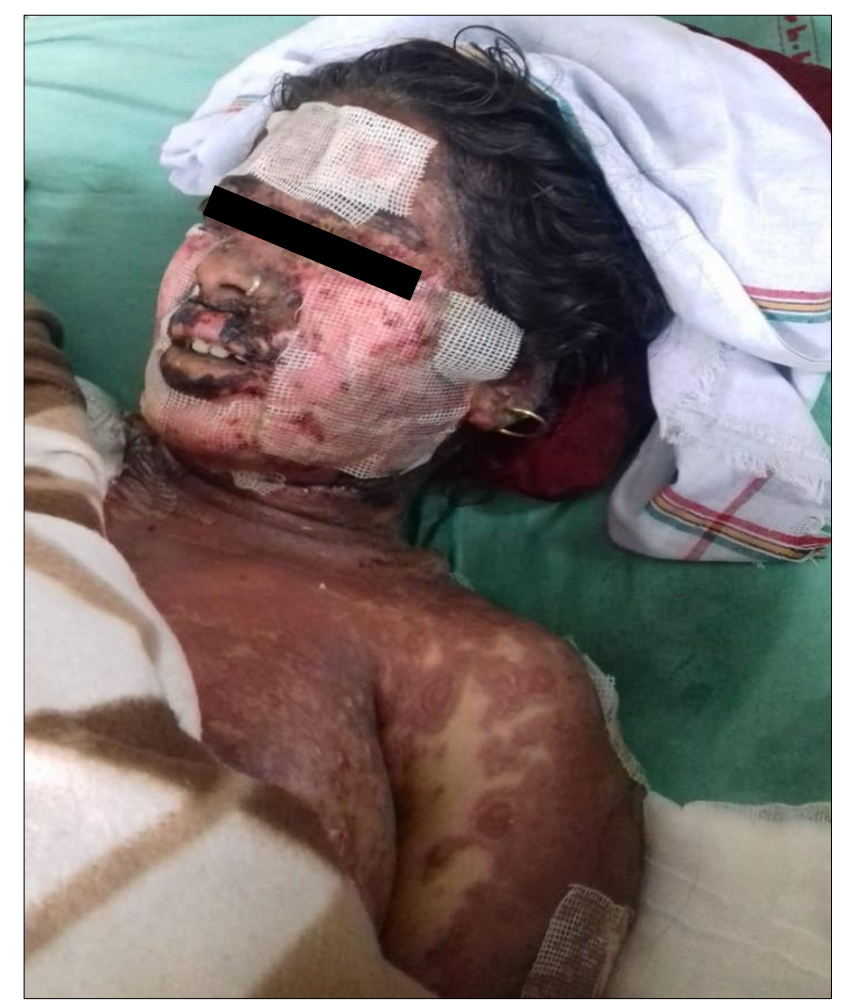

Figure 1: Morbilliform eruptions all over the body.

CNS and cardiorespiratory examination was normal. Abdominal examination revealed a 32 weeks sized uterus with cephalic presentation with a regular fetal heart sound of 132 beats/min. After dermatology referral a provisional diagnosis of viral fever with rashes or drug induced reaction (rashes) was made. Patient was admitted and fever investigation, cultures, skin biopsy were sent. Patient was started on oral acyclovir and oral azithromycin. Calamine lotion was advice for local application.

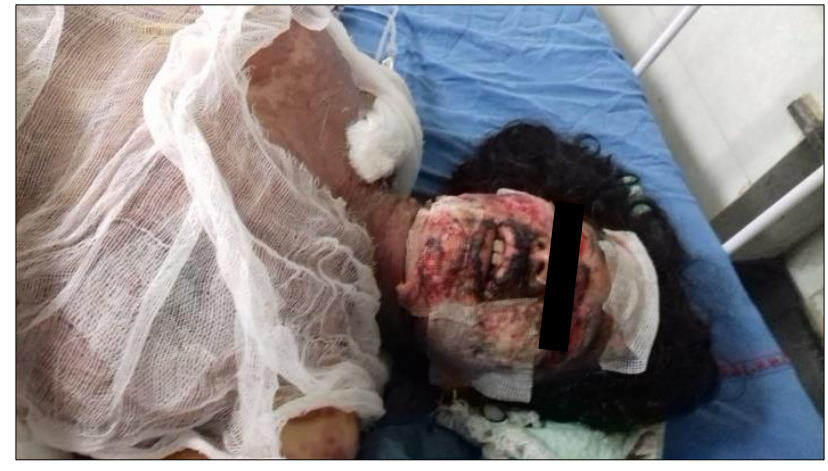

Figure 2: Large blisters covering more than $75 \%$ body surface area.

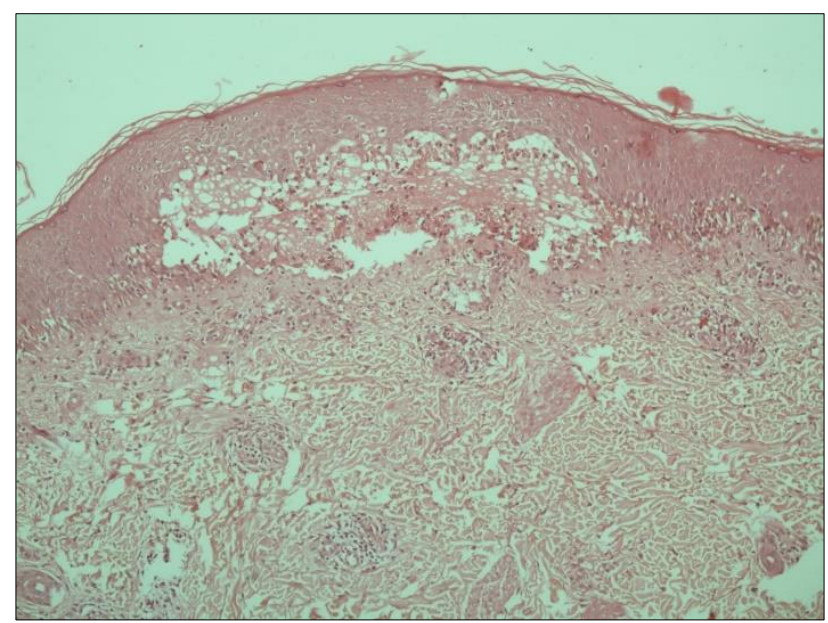

Figure 3: Typical histologic appearance.

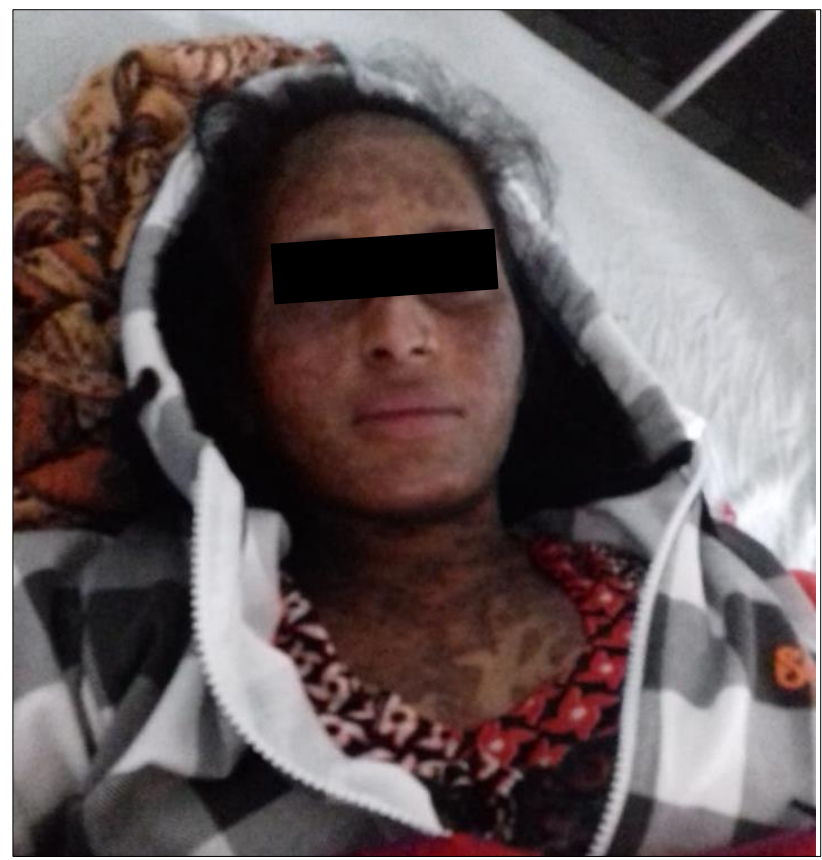

Figure 4: Hypopigmented lesions on recovery.

Initial investigation showed blood group $\mathrm{B}+$, haemoglobin of $10.4 \mathrm{gm} / \mathrm{dl}$, total leucocyte count of 
12,800 , platelet count 1.6 lacs, fasting blood sugar was 78 mg \%, kidney and liver function tests were normal, coagulation profile and urine routine microscopy reports were normal. Obstetric USG report showed SLIUF 31+2 weeks cephalic presentation placenta anterior not lowlying liquor adequate EFW 1533 gms with normal doppler study. Investigation of fever showed positive Widal test.

Over a period of one-week patient's condition deteriorated, new and old mucocutaneouse eruption with large blisters covered more than $75 \%$ of body surface area (Figure 2) and gradually patient had difficulty in eye and mouth opening, had multiple spikes of fever, toxic look and was dehydrated. There was maternal tachycardia, hematuria, increased pallor, edema and swelling all over the body. A multidisciplinary approach (medicine, dermatology, eye, ENT), was sought and patient was provisionally diagnosed as TEN with secondary infection and was put on intravenous acyclovir and $\mathrm{i} / \mathrm{v}$ azithromycin, metrogyl, linzolids. Systemic steroid therapy was started. For analgesia injection paracetamol was continued. Along with intravenous fluid patient was allowed orally. For eye care tobramycin and ciplox eye drop and tear drops were given 3 to 4 times a day. For oral care candid mouth paint, betadine gargle and mucaine gel was used. For blister care blister drainage with intact skin was done and parafiine gauze dressing with $1 \%$ clotrimazole and $2 \%$ muperocin cream was done twice a day.

Skin biopsy report showed TEN and confirmed the diagnosis (Figure 3) and patient was started on cyclosporine $50 \mathrm{mg}$ TDS.

Over the period of one week new blister eruption decreased and old blister started healing but superimposed secondary infection of blisters with sepsis developed as successive blister pus culture sensitivity and blood culture sensitivity report showed acenetobactor infection. For that patient's antibiotics was changed to intravenous colistin and clindamycin. After 8 days patient went into spontaneous labour. She was delivered a live male baby of $1.7 \mathrm{~kg}$ vaginally. Her intrapartum and immediate postpartum period was uneventful.

Over the period of one-month patient condition gradually improved and patient was discharged in satisfactory condition. The only remaining sequelae on follow up was hypo-hyper pigmentation of skin (Figure 4). There was no remaining oral, ocular and genitourinary complication. Baby also had no complication on follow-up.

\section{DISCUSSION}

TEN is a rare life-threatening adverse cutaneous reaction with epidermolysis of more than $30 \%$ TBSA. SJS or toxic epidermal necrolysis (TEN) is one of the dermatologic conditions that can be potentially fatal. ${ }^{5}$ Although the exact etiology of SJS/TEN is not fully understood, it is believed to be an immune mediated hypersensitivity reaction in which cytotoxic T-lymphocytes play a role in the pathogenesis. ${ }^{6}$ Patel et al, reported that penicillins are one of the antimicrobials frequently causing severe cutaneous adverse drug reactions (CADRs) in the Indian population. ${ }^{7}$ Suchi et al, reported a case TEN/SJS in pregnancy precipitated by oral Cefixime. ${ }^{8}$ Similarly, our patient also had history of intake of oral Cefixime before development of TEN. SJS is marked by the rapid attack of fever, skin lesions and sores on the mucous membranes of eyes, mouth, nasal passage, lips and genitals. Clusters last for about 2-4 weeks. The diagnosis is often obvious by the appearance of lesions and rapid progression of symptoms.

Histologic examination of sloughed skin shows necrotic epithelium; a characteristic feature. The condition is fatal and may result in death from pneumonia, septicaemia, myocarditis or renal failure. Severe scarring of the genital tract may also occur occasionally. There has been one case report of vaginal stenosis following SJS in pregnancy, which was discovered 6 weeks after cesarean section for breech presentation. ${ }^{9}$ However, our patient delivered baby vaginally and there was no problem on follow up.

The management includes prompt withdrawal of all potential causative drugs, intravenous fluid replacement.

There have been previous reports of SJS/TEN manifesting in both the mother and the foetus when the disease occurs during pregnancy. ${ }^{10}$ In our case the baby was not affected.

It is difficult to prevent an initial attack of StevensJohnson syndrome because what triggers it is not known. However, if Steven- Johnson syndrome occurred once, which was caused by medication, the drug is to be avoided to prevent another attack. A recurrence is usually more severe than the first episode and, may be fatal.

Attack of SJS developing in pregnancy can be fatal because immunity is compromised.

However, early diagnosis and prompt management saved the mother and the child.

\section{Funding: No funding sources \\ Conflict of interest: None declared \\ Ethical approval: Not required}

\section{REFERENCES}

1. Roujeau JC. Stevens-Johnson syndrome and toxic epidermal necrolysis are severity variants of the same disease which differs from erythema multiforme. J Dermatol. 1997;24:726-9.

2. Knight L, Todd G, Muloiwa R, Matjila M, Lehloenya RJ. Stevens Johnson syndrome and toxic epidermal necrolysis: maternal and foetal outcomes 
in twenty-two consecutive pregnant HIV infected women. PLoS One. 2015;10(8):e0135501.

3. Rodriguez G, Trent JT, Mirzabeigi M, Zaulyanov L, Bruce J, Vincek V. Toxic epidermal necrolysis in a mother and fetus. J Am Acad Dermatol. 2006;55(5)(suppl):S96-S8.

4. Struck MF, Illert T, Liss Y, Bosbach ID, Reichelt B, Steen M. Toxic epidermal necrolysis in pregnancy: case report and review of the literature. J Burn Care Res. 2010;31(5):816-21.

5. Patil JR, Motghare VM, Deshmukh VS, Jaykare SC, Pise HN. Penicillin-induced Steven-Johnson Syndrome a case report. Indian J Clin Pract. 2013;24:626-7.

6. Harr T, French LE. Toxic epidermal necrolysis and Stevens-Johnson syndrome. Orphanet J Rare Dis. 2010;5:39.

7. Patel TK, Barvaliya MJ, Sharma D, Tripathi C. A systematic review of the drug-induced StevensJohnson syndrome and toxic epidermal necrolysis in
Indian population. Indian J Dermatol Venereol Leprol. 2013;79(3):389-98.

8. Jain S, Jain AP, Palaparthy S, Samal S. Steven Johnson syndrome in pregnancy. J MGIMS. 2011;16:48-50.

9. Graham RAC, Cochrane GW, Swihone JR, Sarkany I, Epsztein LJ. Vaginal Stenosis due to Bullous Erythema Multiforme (Steven Johnson Syndrome). Br J Obstet Gynaecol. 1981;88:1156-57.

10. Rodriguez G, Trent JT, Mirzabeigi M, Zaulyanov L, Bruce J, Vincek V. Toxic epidermal necrolysis in a mother and fetus. J Am Acad Dermatol. 2006;55:S96-98.

Cite this article as: Yadav R, Srivastava A, Pawar P, Mendiratta V, Tayal P. A rare case of toxic epidermal necrolysis in pregnancy. Int J Reprod Contracept Obstet Gynecol 2020;9:846-9. 